\title{
A multiplicative Schwarz method with active subdomains for transient convection-diffusion problems
}

\author{
M. L. Sandoval ${ }^{1, *, \dagger}$ and A. Rodríguez-Ferran ${ }^{2}$ \\ ${ }^{1}$ Departamento de Matemáticas, Universidad Autónoma Metropolitana-Iztapalapa, Av. San Rafael Atlixco \#186, \\ Col. Vicentina 09340 Mexico, D.F. \\ ${ }^{2}$ Laboratori de Calcul Numèric (LaCàN), Departament de Matemàtica Aplicada III, E.T.S. d'Enginyers de Camins, \\ Universitat Politècnica de Catalunya, Barcelona, Spain
}

\begin{abstract}
SUMMARY
An efficient algorithm to find the solution of transient convection-diffusion problems with dominant convection is presented. The main idea is to follow the solution front and activate those subdomains where the solution satisfies a given threshold value. We call this novel method 'the multiplicative Schwarz method with active subdomains', and it is motivated by the solution of a problem from activated-carbon filters used in the automotive industry to reduce emissions. Numerical experiments show that this method is more efficient than the preconditioned conjugate gradient method with an incomplete Cholesky factorization.
\end{abstract}

KEY WORDS: multiplicative Schwarz method; active subdomains; symmetric linear systems; convectiondiffusion; activated-carbon filters

\section{INTRODUCTION}

In many convection-diffusion-reaction processes with dominant convection, an abrupt front is formed. This relatively narrow front, which propagates with time due to the transient nature of the process, concentrates all the 'physical activity' and separates two inactive zones. This is the case, for instance, in forest fire spread [1], where the burning front separates the completely burnt zone from the still intact zone.

Another such process is the operation of activated-carbon filters. During loading, the 'front' formed by the hydrocarbon concentration is abrupt and moves as the particles of activated-carbon are saturated [2]. We use a fractional-step method to decouple the convection-diffusion phase (linear) from the reaction phase (non-linear). Since the latter can be handled efficiently at the node level, our analysis is centered in the convection-diffusion phase where the convection is dominant. Following Huerta et al. [3,4], we combine a Crank-Nicolson time-integration scheme with a standard least-squares formulation to stabilize the convective term. The implicit treatment of convective terms usually results in non-symmetric matrices. This is the case for a standard Galerkin approach (i.e. no stabilization) and for several stabilization techniques, such as streamline

\footnotetext{
*Correspondence to: M. L. Sandoval, Departamento de Matemáticas, Universidad Autónoma Metropolitana-Iztapalapa, Av. San Rafael Atlixco \#186, Col. Vicentina 09340 México, D.F.

†'E-mail: mlss@xanum.uam.mx

Contract/grant sponsor: Ministerio de Ciencia e Innovación; contract/grant numbers: CGL2008-06003-C03-02/CLI, DPI2007-62395
} 
upwind Petrov-Galerkin, Galerkin/Least-Squares (GLS) and sub-grid scale (SGS). Least-squares stabilization, on the contrary, leads to a symmetric matrix. Hence, we should solve thousands of linear algebraic systems

$$
\mathbf{A c}^{n+1}=\mathbf{b}
$$

where the matrix A is large, sparse, symmetric, positive definite and constant at each time-step $n$. A finite element discretization described in [5] is applied.

Owing to the formation of an abrupt front, the global solution varies only in a small part of the complete domain at each time-step. Our proposal is to exploit this fact by combining a domain decomposition method with the activation and inactivation of subdomains depending on how the hydrocarbon concentration front moves.

We decided to use the multiplicative Schwarz method (MSM) because it converges faster than the additive Schwarz method [6], and its convergence rate depends on both the overlap size and the number of subdomains $[7,8]$. We want to emphasize that the MSM is used directly as a block Gauss-Seidel solver [9] rather than as a preconditioner.

Since it is important to order the subdomains (and nodes) in the flow direction $[6,10]$, the nodes of the mesh are reordered following the typical block-interface structure of many activated-carbon filters, see [5]. Therefore, a block-tridiagonal global matrix is obtained. In addition, for each block, the reverse Cuthill-McKee algorithm [11] is used to reduce the bandwidth of the matrix.

This article is organized as follows. In Section 2, we define the subdomains that are used in the MSM. In Section 3, we introduce the appropriate criteria to activate or inactivate subdomains. In Section 4, we present our proposal: the MSM with active subdomains. In Section 5, we show some numerical experiments illustrating the performance of the new algorithm, as well as some comparisons with the classical direct and iterative solvers. We close the paper with some concluding remarks.

\section{DEFINITION OF SUBDOMAINS FOR THE MSM}

The region of interest $\bar{\Omega} \subset \mathbb{R}^{3}$ is an activated-carbon filter that is partitioned into $M$ overlapping subdomains $\Omega_{i}, i=1, \ldots, M$, with $\Omega_{i} \cap \Omega_{i+1} \neq \emptyset$ and $\bar{\Omega}=\bigcup_{i=1}^{M} \bar{\Omega}_{i}$, see Figure 1 .

We call 'block', in the context of domain decomposition, to the subsets formed by either $\Omega_{i} \cap$ $\Omega_{i+1}$ (air-chambers) or $\Omega_{i} \backslash\left(\Omega_{i-1} \cup \Omega_{i+1}\right)$ (carbon-chambers), where each block $B_{j}$ is associated with one chamber. With this convection the subdomains next to the filters ends are formed by two blocks and the interior subdomains are formed by three blocks. Overlap always takes place in the air-chambers, see Figure 1, and it is verified that $\bar{\Omega}=\bigcup_{j=1}^{2 M-1} \bar{B}_{j}$.

\section{CRITERIA TO ACTIVATE AND INACTIVATE SUBDOMAINS}

The main difficulty of the proposed method consists in defining appropriately the criteria to activate and inactivate subdomains. The activated-carbon filters are used to expose clearly the criteria.

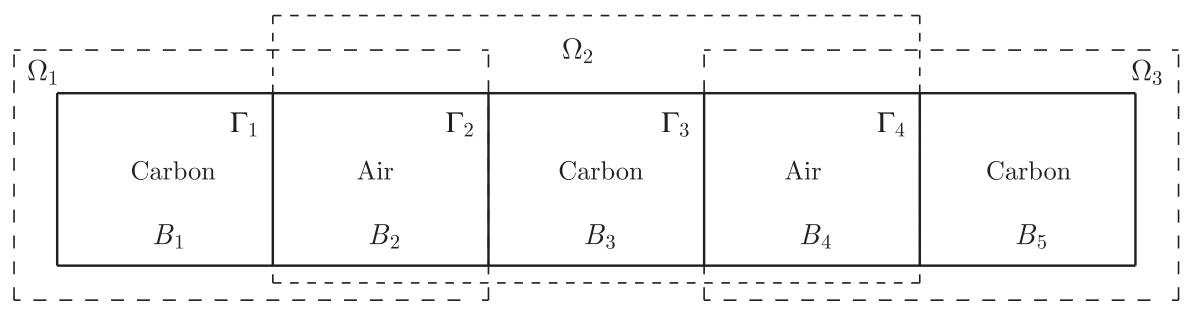

Figure 1. Three overlapping subdomains for a filter with five chambers. 


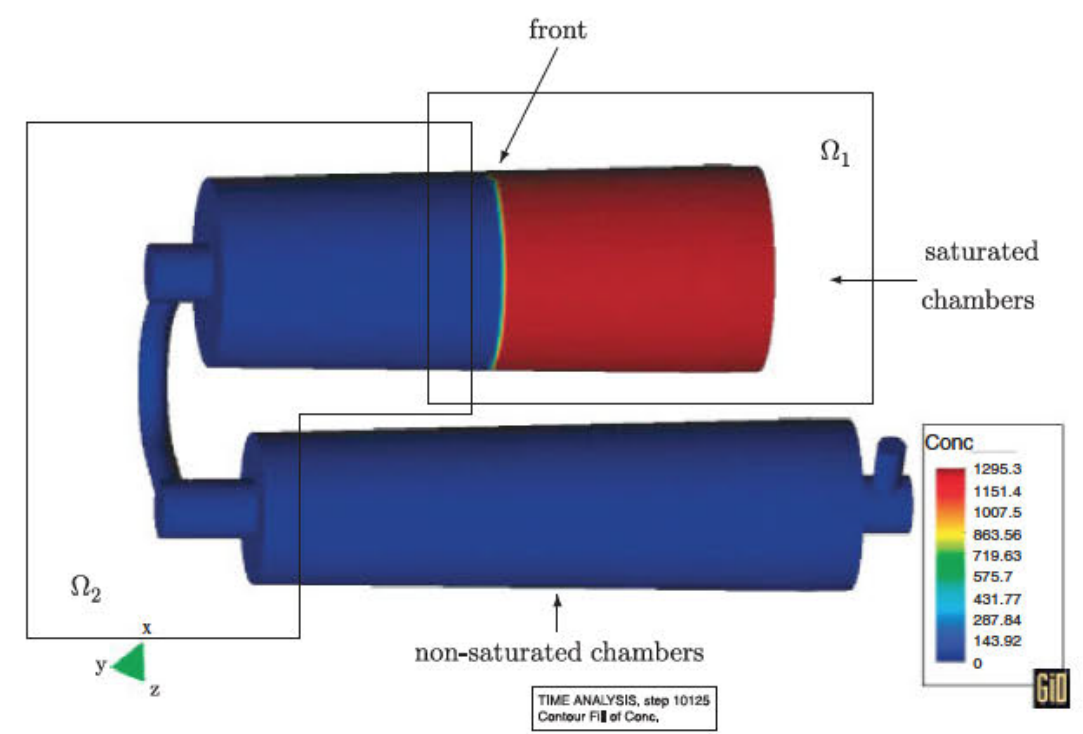

Figure 2. Hydrocarbon front in a load process.

Suppose that the front of fluid has advanced several subdomains. At this point there are two places where the hydrocarbon concentration does not vary: in the saturated subdomains behind the front where the concentration is equal to the external concentration and in the subdomains ahead the front where the concentration is null. We denote by $\Omega_{\text {inac_s }}$ the first set of subdomains and by $\Omega_{\text {inac } \_\emptyset}$ the last set of subdomains, respectively. Let $\Omega_{\text {inac }}$ be the union of inactive subdomains $\Omega_{\text {inac_s }} \cup \Omega_{\text {inac } \_~} \emptyset$ and let $\Omega_{\text {act }}=\Omega \backslash \Omega_{\text {inac }}$ be the set of active subdomains where the hydrocarbon concentration has an effective change. Note that the hydrocarbon concentration varies only in one or two subdomains at each time-step, see Figure 2.

The activation and inactivation is carried out on blocks $B_{j}$ first and later on subdomains $\Omega_{i}$ at each time-step. Two conditions must be satisfied to inactivate a block: (1) the solution satisfies the requirements of precision (residual criterion) and (2) the block is saturated (concentration criterion). These ideas can be described by the following algorithm:

1. Activate and inactivate blocks:

(a) For all $B_{j} \in \Omega_{\text {act }} \cup \Omega_{\text {inac_ø, }}$, compute $\mathbf{r}_{B_{j}}^{n+1}=\mathbf{b}_{B_{j}}-\mathbf{A c}_{B_{j}}^{0, n+1}$.

(b) Activate or inactivate blocks by the residual criterion:

If $\left\|\mathbf{r}_{B_{j}}^{n+1}\right\|_{2}<\operatorname{tol}_{r}\|\mathbf{b}\|_{2}$, then $B_{j} \in \Omega_{\text {inac }}$ else $B_{j} \in \Omega_{\text {act }}$.

(c) Verify that the new active and inactive blocks satisfy the concentration criterion:

- If $B_{j}$ is the most recent inactive block and satisfies

$$
\left\|c_{B_{j}}^{0, n+1}-c_{\mathrm{ext}_{j}}\right\|_{0}>\operatorname{tol}_{c}\left\|c_{\mathrm{ext}}\right\|_{0},
$$
then $B_{j} \in \Omega_{\text {act }}$.

- If the first active block $B_{j}$ satisfies $\left\|c_{B_{j}}^{0, n+1}-c_{\text {ext }_{j}}\right\|_{0} \leqslant \operatorname{tol}_{c}\left\|c_{\text {ext }}\right\|_{0}$, then $B_{j} \in \Omega_{\text {inac }}$.

2. Activate and inactivate subdomains:

(a) If $\Omega_{i}$ contains at least an active block, then $\Omega_{i}$ is activated.

- If $B_{j}$ is an active block in $\Omega_{i} \cap \Omega_{i+1}$, then $\Omega_{i}$ and $\Omega_{i+1}$ are activated.

(b) All subdomains are activated in the first time-step. 
Above, $c_{\text {ext }}$ is the external concentration, $\operatorname{tol}_{c}$ and tol $r$ are tolerances that are prescribed and the norm $\|\cdot\|_{0}$ is defined by $\sqrt{\int_{\Omega} v^{2} \mathrm{~d} \Omega}$.

Note that $\Omega_{\text {inac_s }}$ remains constant or increases at each time-step. Similarly, the set $\Omega_{\text {inac } \_}$ remains constant or decreases at each time-step.

\section{MSM WITH ACTIVE SUBDOMAINS}

Taking everything in the previous sections into account, we propose the MSM with active subdomains, which is described below.

Algorithm of the MSM with active subdomains:

$\mathbf{c}^{0}$ : initial approximation with the solution of the previous time-step

While (non-convergence \& $k<k \max$ )

If $\Omega_{1} \subset \Omega_{\text {act }}$, solve

$$
\left(\begin{array}{cc}
\mathbf{A}_{1,1} & \mathbf{A}_{1,2} \\
\mathbf{A}_{2,1} & \mathbf{A}_{2,2}
\end{array}\right)\left(\begin{array}{c}
\mathbf{c}_{B_{1}}^{k+1} \\
\mathbf{c}_{B_{2}}^{k+1}
\end{array}\right)=\left(\begin{array}{c}
\mathbf{b}_{B_{1}} \\
\mathbf{b}_{B_{2}}-\mathbf{A}_{2,3} \mathbf{c}_{B_{3}}^{k}
\end{array}\right)
$$

For each $i=2, M-1$ and $\Omega_{i} \subset \Omega_{\text {act }}$,

solve

$$
\left(\begin{array}{ccc}
\mathbf{A}_{2 i-2,2 i-2} & \mathbf{A}_{2 i-2,2 i-1} & \mathbf{0} \\
\mathbf{A}_{2 i-1,2 i-2} & \mathbf{A}_{2 i-1,2 i-1} & \mathbf{A}_{2 i-1,2 i} \\
\mathbf{0} & \mathbf{A}_{2 i, 2 i-1} & \mathbf{A}_{2 i, 2 i}
\end{array}\right)\left(\begin{array}{c}
\mathbf{c}_{B_{2 i-2}}^{k+1} \\
\mathbf{c}_{B_{2 i-1}}^{k+1} \\
\mathbf{c}_{B_{2 i}}^{k+1}
\end{array}\right)=\left(\begin{array}{c}
\mathbf{b}_{B_{2 i-2}}-\mathbf{A}_{2 i-2,2 i-3} \mathbf{c}_{B_{2 i-3}+1}^{k+1} \\
\mathbf{b}_{B_{2 i-1}} \\
\mathbf{b}_{B_{2 i}}-\mathbf{A}_{2 i, 2 i+1} \mathbf{c}_{B_{2 i+1}}^{k}
\end{array}\right)
$$

End $i$

If $\Omega_{M} \subset \Omega_{\text {act }}$, solve

$$
\left(\begin{array}{ll}
\mathbf{A}_{2 M-2,2 M-2} & \mathbf{A}_{2 M-2,2 M-1} \\
\mathbf{A}_{2 M-1,2 M-2} & \mathbf{A}_{2 M-1,2 M-1}
\end{array}\right)\left(\begin{array}{c}
\mathbf{c}_{B_{2 M-2}}^{k+1} \\
\mathbf{c}_{B_{2 M-1}}^{k+1}
\end{array}\right)=\left(\begin{array}{c}
\mathbf{b}_{B_{2 M-2}}-\mathbf{A}_{2 M-2,2 M-3} \mathbf{c}_{B_{2 M-3}}^{k+1} \\
\mathbf{b}_{B_{2 M-1}}
\end{array}\right)
$$

End $k$

Note that: (1) iterations are initialized with the solution of the previous time-step $\left(\mathbf{c}^{0, n+1}=\mathbf{c}^{n}\right)$, (2) for $i=1, \ldots, M$, blocks $\Omega_{i} \cap \Omega_{i+1}$ and $\Omega_{i} \backslash\left(\Omega_{i-1} \cup \Omega_{i+1}\right)$ are associated to indices $2 i$ and $2 i-1$, respectively and (3) $\mathbf{c}^{k, n+1}=\mathbf{c}^{k}$ is used to simplify the notation.

We emphasize that 'active subdomain' means that its associated subproblem has been solved. We also emphasize that $\Omega=\Omega_{\text {inac_s }} \cup \Omega_{\text {act }} \cup \Omega_{\text {inac } \_\varnothing}$ induces automatically a partition in the concentration vector: $\mathbf{c}=\left(\mathbf{c}_{\mathrm{ext}}, \mathbf{c}_{\mathrm{act}}, \mathbf{0}\right)^{\mathrm{T}}$.

\subsection{Subsystems solution: direct and iterative methods}

The linear subsystems of equations can be solved by either direct or iterative methods; the latter are typically more efficient, see for instance [10,12-15]. However, we use both approaches to find the solution of the subsystems. Since the matrices associated to the subsystems are principal submatrices of the global matrix $\mathbf{A}$, they are symmetric and positive definite [16]. Hence, the linear subsystems are solved by either: (1) the Cholesky factorization [17] or (2) the preconditioned conjugate gradient method [11] with the drop-tolerance incomplete Cholesky factorizations [18], which are efficient for transient convection-diffusion problems according to the numerical results obtained in [5]. 


\section{NUMERICAL EXPERIMENTS}

The main goal of this section is to discuss the computational performance of the MSM with active subdomains for transient convection-diffusion problems. We compare both memory requirements and central processing unit (CPU) time with three different approaches: the Cholesky factorization (without iterations), the Cholesky factorization with iterative refinement [19] and the incomplete Cholesky conjugate gradient method (ICCG) with threshold $\tau=5 \times 10^{-3}$, see [5]. Before we do the comparison, we verify the criteria to activate and inactivate subdomains and some properties that are preserved from the MSM.

In order to simplify the exposition we introduce the following notation:

- Chol, Cholesky factorization,

- IR, iterative refinement method,

- $\operatorname{ICCG}(\tau)$, incomplete Cholesky conjugate gradient method with threshold $\tau$ [18],

- DD-Chol, MSM with active subdomains using Cholesky factorization,

- $\operatorname{DD}-\mathrm{IC}(\tau), \mathrm{MSM}$ with active subdomains using $\operatorname{ICCG}(\tau)$,

- A, global matrix,

- $\mathbf{A}_{\mathbf{D}}$, block-diagonal matrix formed by the submatrices of the MSM with active subdomains algorithm.

We work basically with two filters, denoted by D and E, see Figure 3. For filter D, we use three unstructured meshes: 'coarse', 'fine' and 'extra fine'. We also consider a 'coarse' mesh for the
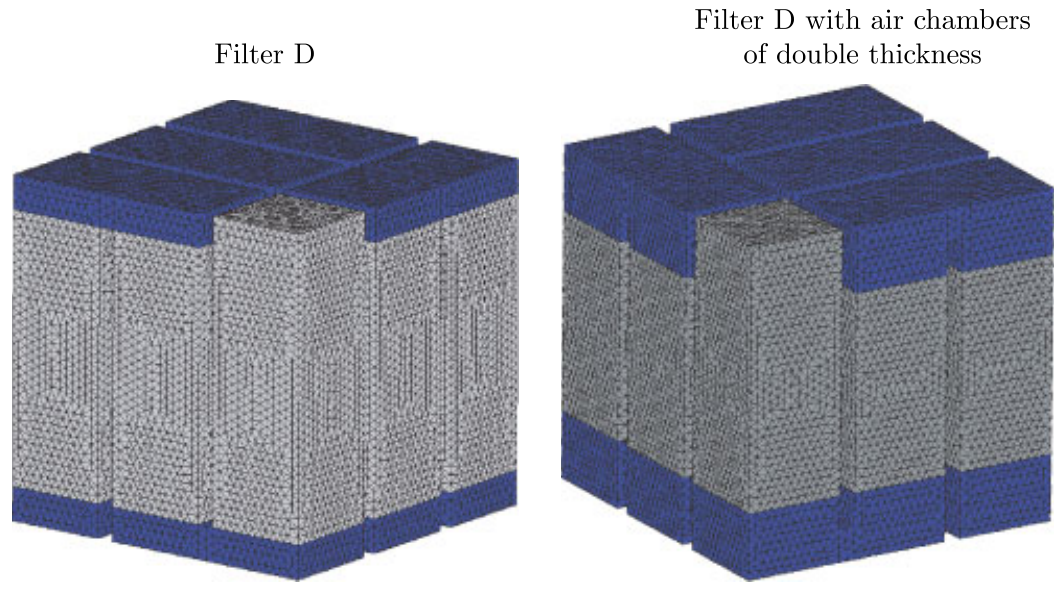

Filter E

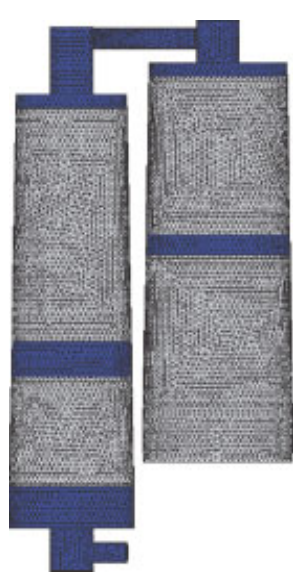

Figure 3. Activated-carbon filters, coarse meshes. 
Table I. Main numerical parameters in filters simulations.

\begin{tabular}{|c|c|c|c|c|c|c|}
\hline & \multicolumn{4}{|c|}{ Filter D } & \multicolumn{2}{|c|}{ Filter E } \\
\hline & Coarse & Fine & Extra fine & Double thickness & Coarse & Fine \\
\hline \# nodes & 87703 & 141706 & 591857 & 86624 & 87970 & 171802 \\
\hline \# elements & 453174 & 747641 & 3274220 & 447228 & 472277 & 940695 \\
\hline$N$ & 87527 & 141465 & 591261 & 86448 & 87274 & 170850 \\
\hline $\mathrm{nnz}(\mathbf{A})$ & 648577 & 1059448 & 4532527 & 640569 & 659253 & 1301816 \\
\hline $\operatorname{nnz}\left(\mathbf{A}_{\mathbf{D}}\right)$ & 751912 & 1235555 & 5324074 & 852365 & 729294 & 1435060 \\
\hline \# steps & 81513 & 96272 & 155912 & 64288 & 28961 & 32493 \\
\hline$\Delta t$ & 2.00 & 1.69 & 1.04 & 2.00 & 3.12 & 2.8 \\
\hline$M$ & 9 & 9 & 9 & 9 & 4 & 4 \\
\hline
\end{tabular}

filter D with double thickness of the air chambers (i.e. duplicated overlap). Finally, two meshes are used for filter E: 'coarse' and 'fine'.

For each filter and mesh, Table I summarizes the main numerical parameters: the number of nodes (\# nodes), the number of finite elements (\# elements), the size $N$ of matrix $\mathbf{A}$, the number of non-zero elements $\operatorname{nnz}(\mathbf{A})$ in the upper triangle matrix $\mathbf{A}$, the number of non-zero elements $n n z\left(\mathbf{A}_{\mathbf{D}}\right)$ in the upper triangle block-diagonal matrix $\mathbf{A}_{\mathbf{D}}$, the time-step $\Delta t$, the number of time-steps (\# steps) and the number of subdomains $M$.

All codes are implemented in Fortran 90 with double precision and compiled with the option -xP -O2 -ipo -fixed -tpp7. The numerical experiments were run on a Pentium Xeon EM64T (3.3 GHz) processor with $8 \mathrm{Mb}$ of cache L3. The stopping criteria for both iterative methods are

$$
\begin{aligned}
\left|x_{i}^{k}-x_{i}^{k+1}\right| & \leqslant \operatorname{tol}_{x}\left(\left|x_{i}^{k+1}\right|+1\right) \quad \text { for each component } i \\
\left\|\mathbf{b}-\mathbf{A x} \mathbf{x}^{k+1}\right\|_{2} & \leqslant \operatorname{tol}_{r} \| \mathbf{b}_{2}
\end{aligned}
$$

with tol ${ }_{x}=0.5 \times 10^{-10}$ and tol $_{r}=0.5 \times 10^{-9}$. In addition, for the concentration criterion we use the value tol ${ }_{c}=0.5 \times 10^{-7}$.

\subsection{Verification of criteria to activate and inactivate subdomains}

We present numerical results for filters $\mathrm{D}$ and $\mathrm{E}$ when coarse meshes are used. These results are used to verify the criteria to activate and inactivate subdomains. Figures 4(a), 4(b) and 5(a) illustrate the relative variation of $c_{B_{j}}^{n}$ with respect to $c_{\text {ext }}$ versus time (given by the number of time-steps). In the same way, Figures 4(c) and 5(b) illustrate how each subdomain $\Omega_{i}$ is activated or inactivated. We can interpret these figures in the following way: blocks 2, 3 and 4 in Figure 5(a) form subdomain 2 ; at $n=8025$ the concentration of block 2 is different from zero, so that subdomain 2 is activated (see Figure 5(b)); at $n=19530$ blocks 2, 3 and 4 satisfy the concentration criterion, and therefore subdomain 2 is inactivated.

Note from Figures 4(c) and 5(b) that the process to activate and inactivate subdomains is carried out correctly. Observe also that only two subproblems are solved in a significant percentage of time-steps. In Section 5.3.2 we show that, in spite of this, good numerical results are obtained.

\subsection{Effect of overlap size versus element size}

We now verify numerically that MSM with active subdomains preserves some properties of the standard MSM. Figures 6(a), (b) and (c) include graphs of number of iterations versus number of time-steps for filter $\mathrm{D}$ when the proposed algorithm is used for different meshes and single overlap size. The average number of iterations is shown with a dashed line in every graph. Note that only one iteration is needed for time-steps with just one active subdomain, while a few iterations are required for time-steps with two active subdomains (cf. Figures 6(a) and 4(c)). Figure 6(d) shows the correspondent graph for a coarse mesh with duplicated overlap. Figures 6(a), (b) and (c) show that, as the mesh is refined, the average number of iterations per time-step decreases. Figures 6(a) 

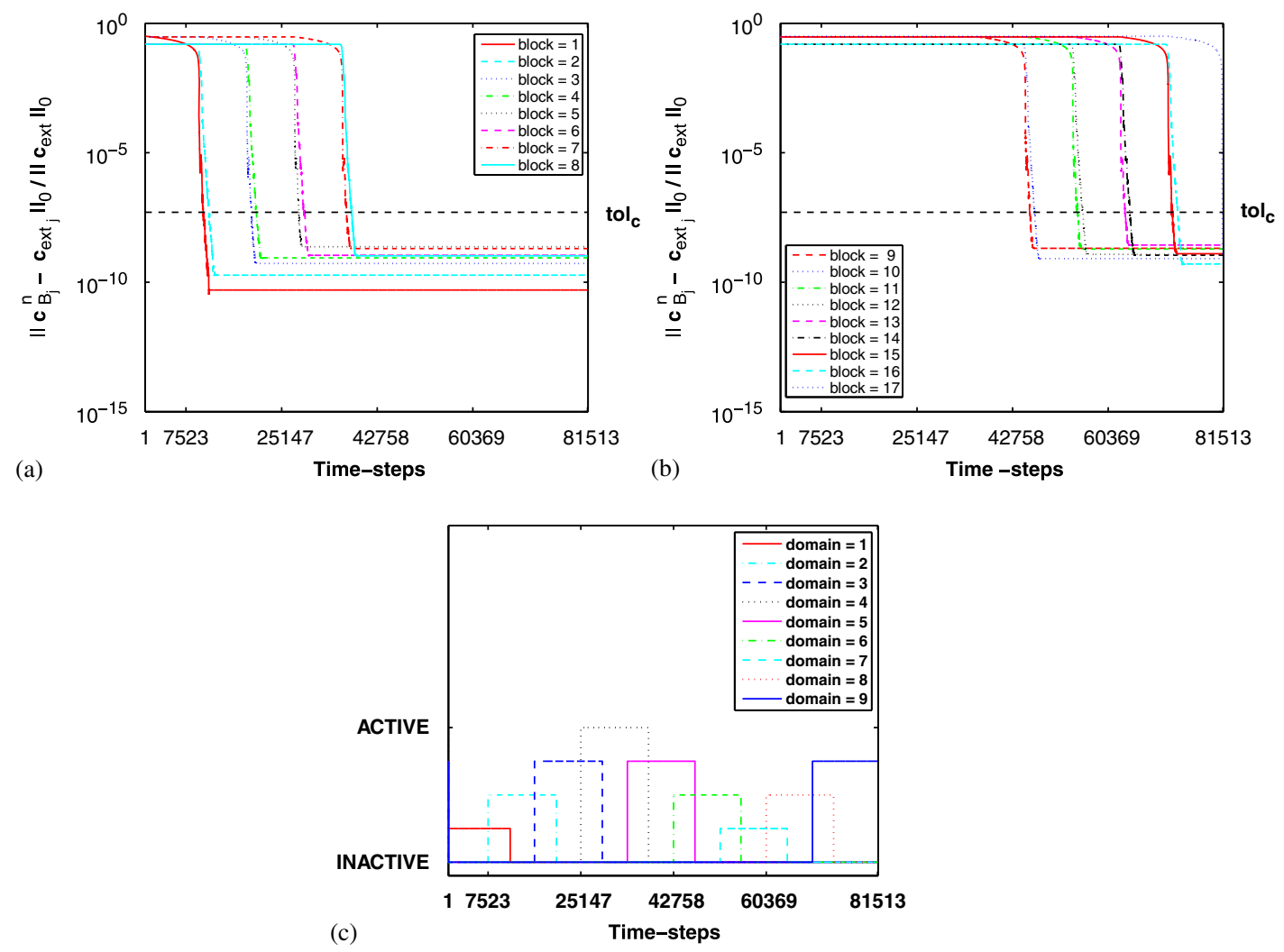

Figure 4. Filter D, coarse mesh: (a) and (b) the relative variation of $c_{B_{j}}^{n}$ with respect to $\mathbf{c}_{\text {ext }}$ versus time-steps and (c) active and inactive subdomains versus time-steps.
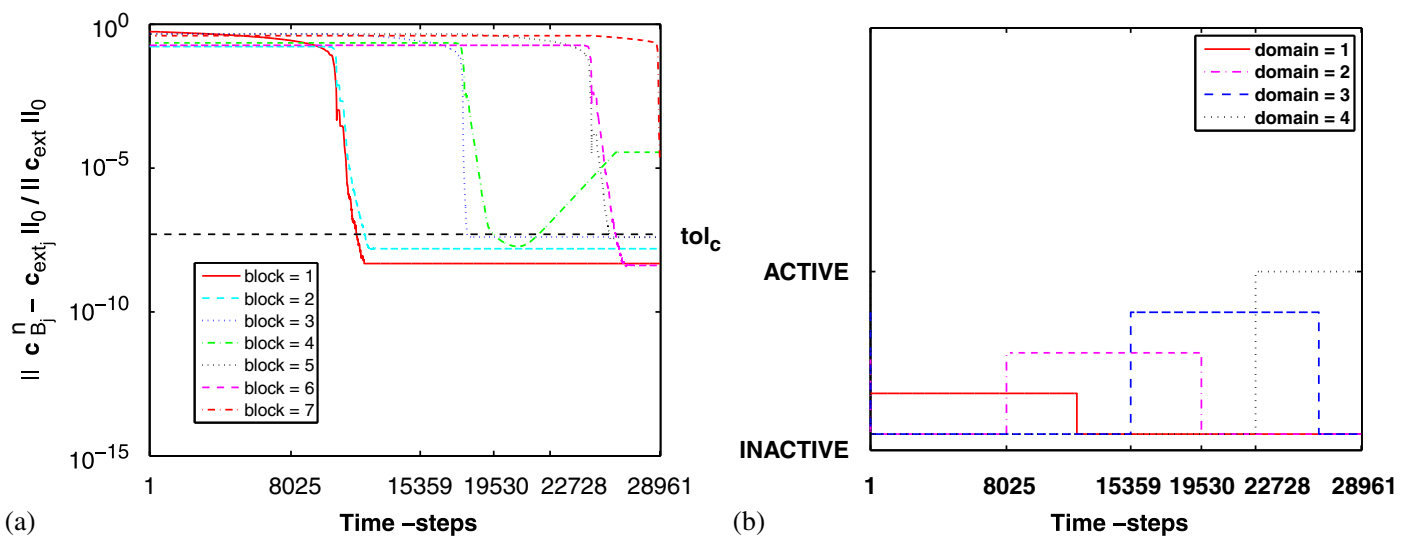

Figure 5. Filter E, coarse mesh: (a) the relative variation of $c_{B_{j}}^{n}$ with respect to $c_{\text {ext }}$ versus time-steps and (b) active and inactive subdomains versus time-steps.

and (d) show that, as overlap size increases, the convergence is faster; the average of iterations decreases from 2.12 to 1.06 . Therefore, MSM with active subdomains preserves some properties of the convergence estimate for the MSM given in [7].

\subsection{Computational performance of MSM with active subdomains}

In order to verify the effectiveness of the proposed algorithm and enforce the direct method to have the same precision, we choose an iterative refinement (IR) algorithm [19]. We take this 

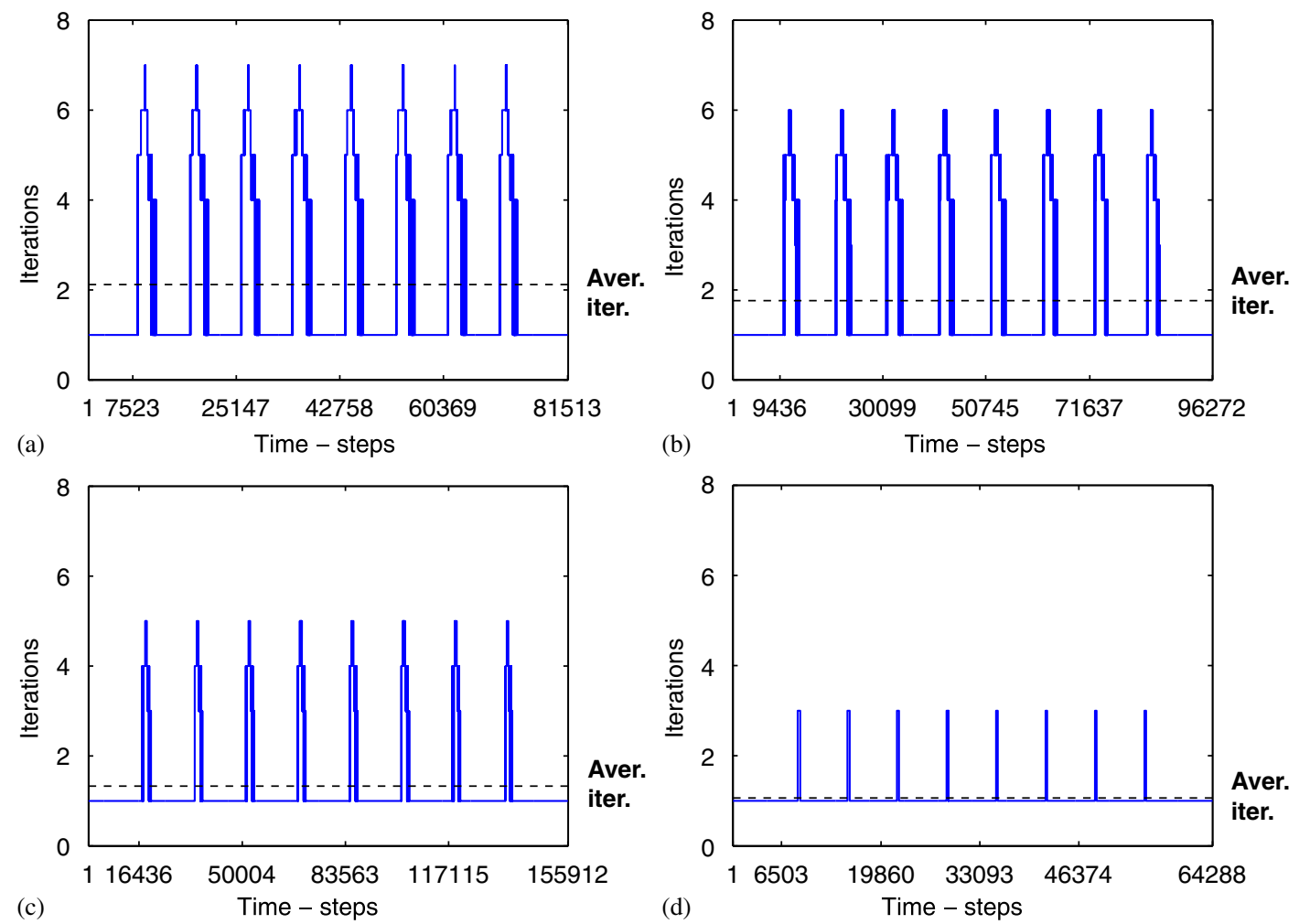

Figure 6. Graphs of the number of iterations versus time-steps (solid line) using DD-Chol for: (a) coarse mesh; (b) fine mesh; (c) extra fine mesh; and (d) coarse mesh with duplicated overlap. Dashed line shows the average number of iterations in every graph.

algorithm as a reference to compare computer memory and CPU time to calculate norms, indexes, new independent vectors, as well as the number of iterations generated by MSM with active subdomains. Table II summarizes the results obtained for filter D when the coarse mesh is used. We can appreciate the relevance of the proposed algorithm since we only require about $13 \%$ more memory to save $45 \%$ of CPU time approximately.

We point out that the additional cost to apply the criteria to activate and inactivate subdomains is compensated by the fact that we only have to solve one or two subdomain problems at each time-step.

Note also that the number of iterations for DD-Chol is duplicated, but the CPU time to compute the residual norm only increases by $46 \%$, since it is only calculated on active subdomains (i.e. $\left.\left\|\mathbf{b}-\mathbf{A c}^{k}\right\|_{2}=\left\|\mathbf{b}_{\text {act }}-\mathbf{A} \mathbf{c}_{\text {act }}^{k}\right\|_{2}\right)$.

Table II also indicates that the iterative refinement is not actually performed: the solution with the direct method already satisfies the accuracy required by the iterative method.

5.3.1. Computational efficiency for the linear subsystems. We compare two strategies to solve the subsystems of linear equations: the Cholesky factorization and the ICCG method. For the ICCG we consider the case of no fill-in and the case with different drop thresholds.

Figure 7 shows the computational cost of the numerical experiments for filters D and E with both meshes, coarse and fine, and Table III summarizes the results for filter E. This table shows: the number of time-steps, the cumulative number of iterations for MSM with active subdomains, the average of iterations for MSM with active subdomains, the cumulative number of iterations to solve the subsystems with ICCG, the average of iterations for ICCG, the average of non-zero elements for the factor $\mathbf{L}$ of the upper triangle block-diagonal matrix $\mathbf{A}_{\mathbf{D}}$ and the required CPU times to calculate $\mathbf{L}$ and to solve the linear systems over all time-steps, also the total CPU time. 
Table II. Filter D, coarse mesh. CPU time for the iterative refinement algorithm and MSM with active subdomains. Boldface numbers are cited in text.

\begin{tabular}{|c|c|c|c|c|c|}
\hline & \multicolumn{2}{|c|}{ IR } & \multicolumn{2}{|c|}{ DD-Chol } & \multirow{2}{*}{$\frac{(\mathrm{DD}-\mathrm{Chol}-\mathrm{IR}) / \mathrm{IR}}{\%}$} \\
\hline & Time (s) & $\%$ & Time (s) & $\%$ & \\
\hline Calculation of $\mathbf{A}$ and $\mathbf{A}_{\mathbf{D}}$ & 2.83 & 0.01 & 2.93 & 0.02 & 4 \\
\hline Factorization & 3.98 & 0.02 & 4.44 & 0.03 & 12 \\
\hline Calculation of $\mathbf{b}$ & 1620.29 & 6.43 & 1637.03 & 11.76 & 1 \\
\hline$\left\|\mathbf{b}-\mathbf{A} \mathbf{c}^{k}\right\|_{2}$ & 782.50 & 3.11 & 1138.66 & 8.18 & 46 \\
\hline Calculation of $\mathbf{b}_{B_{j}}$ & - & - & 151.95 & 1.09 & - \\
\hline$\left\|\mathbf{b}_{B_{j}}-\mathbf{A} \mathbf{c}_{B_{j}}^{0}\right\|_{2}$ & - & - & 11.47 & 0.08 & - \\
\hline Indexes of activation & - & - & 5.44 & 0.04 & - \\
\hline$\left\|c_{B_{j}}^{0}-c_{\mathrm{ext}_{j}}\right\|_{0}$ & - & - & 742.07 & 5.33 & - \\
\hline Additional calculations & 358.74 & 1.42 & 934.88 & 6.72 & 161 \\
\hline Solution & 22107.05 & 87.77 & 8973.54 & 64.49 & -59 \\
\hline Total & 25188.51 & 100 & 13914.76 & 100 & -45 \\
\hline Memory factorization & 23074479 & 100 & 26014881 & 99.66 & 13 \\
\hline Mem. auxiliary vectors & - & - & 87703 & 0.34 & - \\
\hline Total & 23074478 & 100 & 26102584 & 100 & 13 \\
\hline \# time-steps & \multicolumn{2}{|c|}{81513} & \multicolumn{2}{|c|}{81513} & - \\
\hline \# iterations & \multicolumn{2}{|c|}{81513} & \multicolumn{2}{|c|}{172905} & 112 \\
\hline iter/time-steps & \multicolumn{2}{|c|}{1} & \multicolumn{2}{|c|}{2.12} & 112 \\
\hline
\end{tabular}
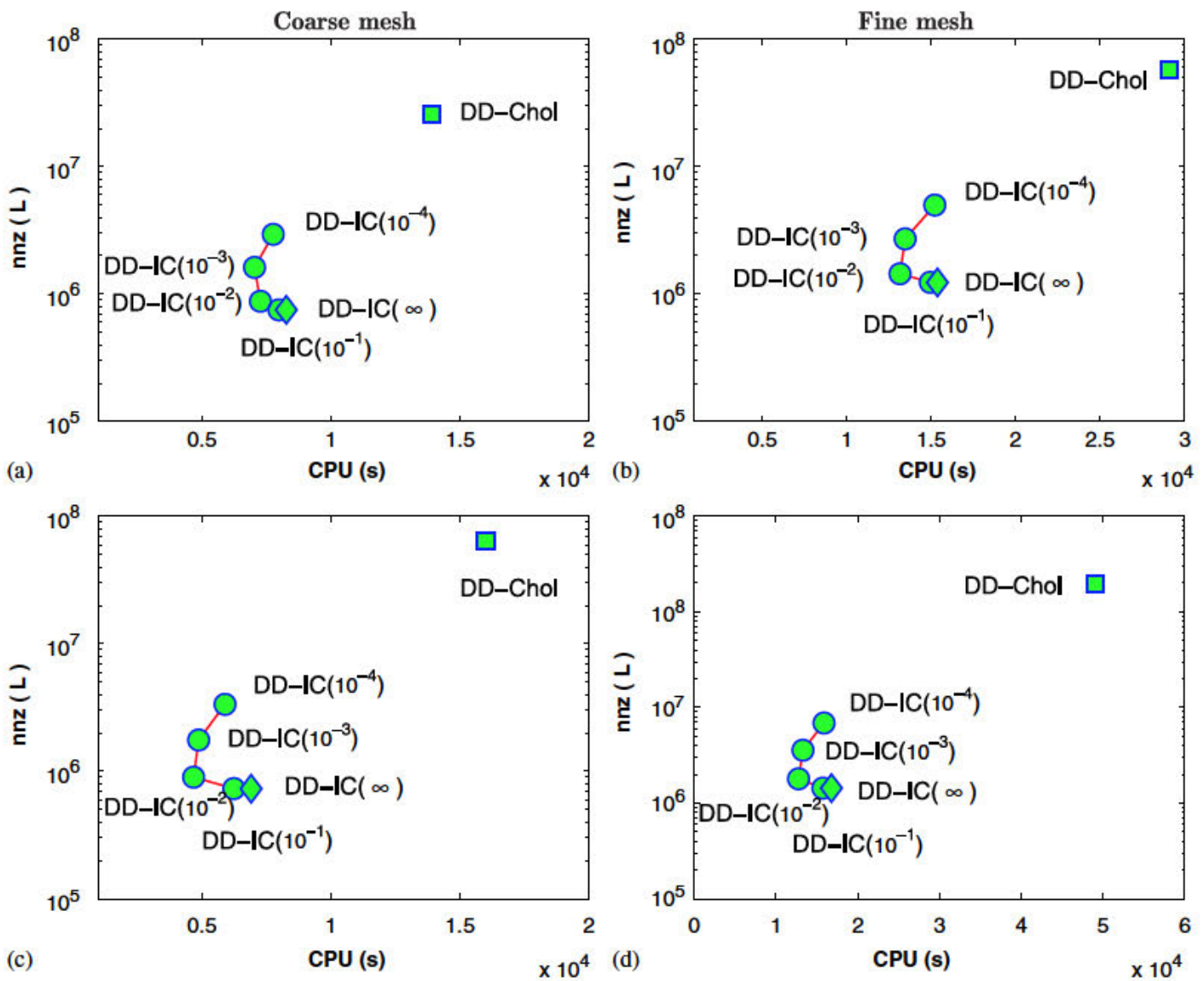

Figure 7. Computational cost of MSM with active subdomains using Cholesky and ICCG solvers for filter D: (a) coarse mesh and (b) fine mesh; and filter E: (c) coarse mesh and (d) fine mesh. 


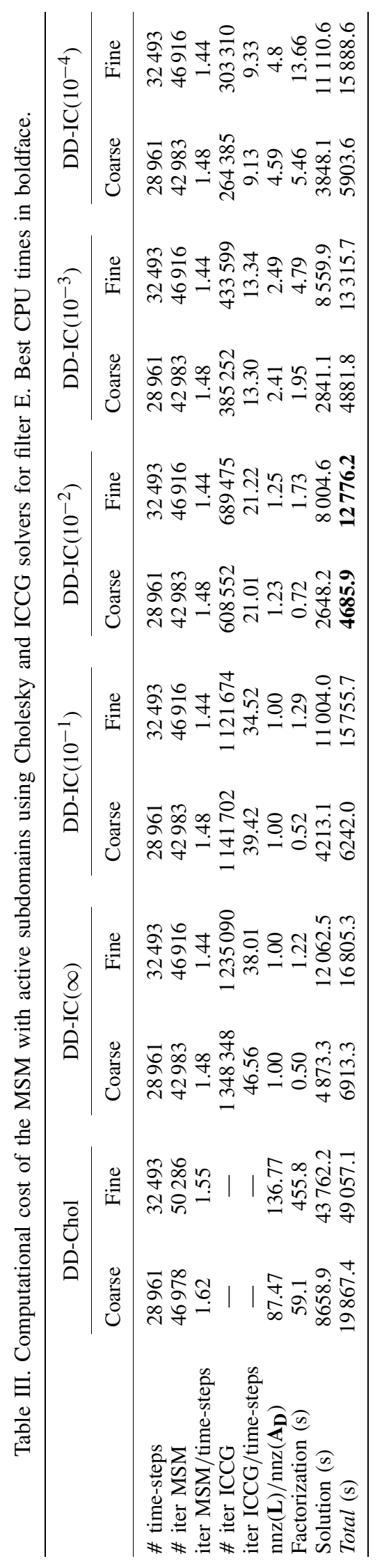



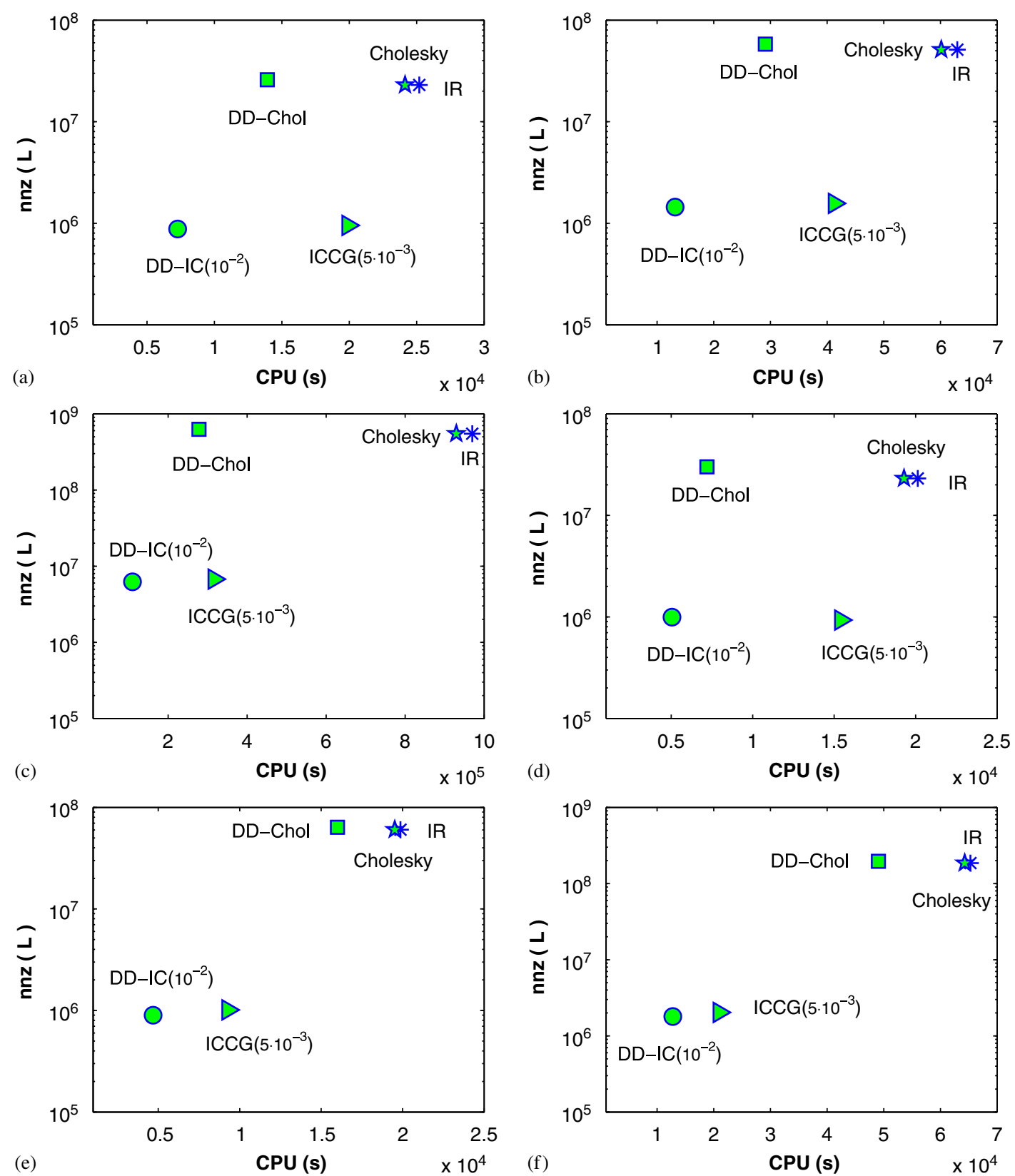

Figure 8. Computational cost of Cholesky, ICCG and MSM with active subdomains solvers for filter D: (a) coarse mesh; (b) fine mesh; (c) extra fine mesh; and (d) coarse mesh with duplicated overlap; and for filter E: (e) coarse mesh and (f) fine mesh.

From the results shown in Figure 7 and Table III we can say that

- MSM with active subdomains requires, on the average, less than two iterations to converge.

- For each time-step, ICCG needs tens of iterations to obtain the solution in all active subdomains.

- The convergence of DD-IC $(\tau)$ is slightly faster than the convergence of DD-Chol: the error when the subproblems are solved with the Cholesky factorization is slightly larger than the required tolerance for the iterative methods.

- IC even with no fill-in reduces CPU time considerably. 
- Allowing a suitable fill-in for $\operatorname{IC}\left(\tau=10^{-2}\right)$, an additional substantial reduction in total CPU time is obtained (for a factor of about 4 ).

- The time needed to compute either complete or incomplete factorization of $\mathbf{A}_{\mathbf{D}}$ is negligible compared with the time required to solve the active subsystems over all time-steps. This result agrees very well with that obtained in [5].

5.3.2. Efficiency of MSM with active subdomains versus solution of the global problem. We now compare the best result that was obtained in the previous section (DD-IC $\left(10^{-2}\right)$ ) with the best result reported in [5] when ICCG $\left(\tau=5 \times 10^{-3}\right)$ is used to solve the global problem (i.e. without domain decomposition). We also use Cholesky factorization and the iterative refinement algorithm. Figure 8 shows the results of these approaches for all the experiments described in Table I. From this figure, we can say that

- If the solution is obtained by DD-IC $\left(10^{-2}\right)$, small overlaps are compensated. The computational costs of cases (a) and (d) are practically the same if $\tau=10^{-2}$.

- As the mesh is refined, saving in CPU time increases drastically: for filter D with the extra fine mesh, Cholesky factorization spends 10.8 days, $\operatorname{ICCG}\left(5 \times 10^{-3}\right)$ takes 3.7 days, while DD-IC $\left(10^{-2}\right)$ requires only 1.3 days.

- In all cases, MSM with active subdomains using ICCG is more efficient. From the previous results it is recommended to use $\tau \in\left[10^{-3}, 10^{-1}\right]$.

\section{SUMMARY AND CONCLUSIONS}

We developed an alternative method called the MSM with active subdomains, which is designed to solve transient convection-diffusion problems where the solution usually has an abrupt front and only changes in a small region of the complete domain at each time-step.

Numerical experiments show that the MSM with active subdomains is more efficient when the subproblems are solved with a drop-tolerance ICCG method than solving the global problem with ICCG alone. However, it would be interesting to compare the performance of different techniques for the solution of subdomain problems, for instance the best $\operatorname{ICCG}(\tau)$ against the multigrid methods, see [15].

According to our results, the threshold $\tau$ should be selected in the interval $\left[10^{-3}, 10^{-1}\right]$. Particularly, we found that $\tau=10^{-2}$ is the best option for the activated-carbon filters. The results also indicate that the computational cost needed to compute the Cholesky decomposition, associated with the subproblems, is insignificant compared with the time to do the iterations. These conclusions agree with those in [5].

Since the numerical efficiency is totally controlled by the application phase of each subproblem at each time-step, using $\operatorname{ICCG}(\tau)$ compensates the fact that more iterations are needed in small overlaps. Therefore, the proposed method seems to be very attractive to solve transient convectiondiffusion problems with fine meshes and different overlaps using a single processor.

Finally, it is important to mention that the proposed criteria to activate and inactivate subdomains can be adapted to different convection-diffusion problems where the unknown is a physical quantity that may be different to concentration of hydrocarbon.

\section{REFERENCES}

1. Ferragut L, Asensio MI, Monedero S. Modelling radiation and moisture content in fire spread. Communications in Numerical Methods in Engineering 2007; 23(9):819-833.

2. Pérez-Foguet A, Huerta A. Mathematical modelling of mass transport equations in fixed-bed absorbes. In Progress in Industrial Mathematics to ECMI 2004, Di Bucchianico A, Mattheij RMM, Peletier MA (eds). Springer: Berlin, 2006.

3. Huerta A, Donea J. Time-accurate solution of stabilized convection-diffusion-reaction equations: I-time and space discretization. Communications in Numerical Methods in Engineering 2002; 18(8):565-573.

4. Huerta A, Roig B, Donea J. Time-accurate solution of stabilized convection-diffusion-reaction equations: IIaccuracy analysis and examples. Communications in Numerical Methods in Engineering 2002; 18(8):575-584. 
5. Rodríguez-Ferran A, Sandoval ML. Numerical performance of incomplete factorizations for 3D transient convection-diffusion problems. Advances in Engineering Software 2007; 38(6):439-450.

6. Xiao-Chuan C, Sarkis M. Local multiplicative Schwarz algorithms for steady and unsteady convection-diffusion equations. East-West Journal of Numerical Mathematics 1998; 6(1):27-41.

7. Bramble JH, Pasciak JE, Wang J, Xu J. Convergence estimates for product iterative methods with applications to domain decomposition. Mathematics of Computation 1991; 57(195):1-21.

8. Widlund O. A comparison of some domain decomposition and iterative refinement algorithms for elliptic finite element problems. Technical Report BSC 88/15, IBM Bergen Scientific Centre, Bergen, Norway, 1988.

9. Tang W-P. Generalized Schwarz splittings. SIAM Journal on Scientific Computing 1992; 13(2):573-595.

10. Cai X-C, Saad Y. Overlapping domain decomposition algorithms for general sparse matrices. Numerical Linear Algebra with Applications 1996; 3(3):221-237.

11. Saad Y. Iterative Methods for Sparse Linear Systems. Society for Industrial and Applied Mathematics: Philadelphia, 2003.

12. Cai X-C, Gropp D, Keyes DE. A comparison of some domain decomposition algorithms and ILU preconditioned iterative methods for nonsymmetric elliptic problems. Numerical Linear Algebra with Applications 1994; 1(5): 477-504.

13. Brakkee E, Vuik C, Wesseling P. Domain decomposition for the incompressible Navier-Stokes equations: solving subdomain problems accurately and inaccurately. International Journal for Numerical Methods in Fluids 1998; 26(10):1217-1238.

14. Garbey M, Tromeur-Dervout D. On some Aitken-like acceleration of the Schwarz method. International Journal for Numerical Methods in Fluids 2002; 40(12):1493-1513.

15. Singh KM, Williams JJR. Application of the additive Schwarz method to large scale Poisson problems. Communications in Numerical Methods in Engineering 2004; 20(3):193-205.

16. Hackbusch W. Iterative Solution of Large Sparse Systems of Equations, vol. 95. Applied Mathematical Sciences. Springer: New York, 1994.

17. Duff IS, Erisman AM, Reid JK. Direct Methods for Sparse Matrices (2nd edn). Oxford University Press: Oxford, 1992.

18. Munksgaard N. Solving sparse symetric sets of linear equations by preconditioned conjugate gradients. ACM Transactions on Mathematical Software 1980; 6(2):206-219.

19. Meurant G. Computer Solution of Large Linear Systems. Studies in Mathematics and its Applications, vol. 28. Elsevier: Amsterdam, 1999. 